\title{
Unlimited Vocabulary Grapheme to Phoneme Conversion for Korean TTS
}

\author{
Byeongchang Kim and WonIl Lee and Geunbae Lee and Jong-Hyeok Lee \\ Department of Computer Science \& Engineering \\ Pohang University of Science \& Technology \\ Pohang, Korea \\ \{bckim, bdragon, gblee, jhlee\}@postech.ac.kr
}

\begin{abstract}
This paper describes a grapheme-to-phoneme conversion method using phoneme connectivity and CCV conversion rules. The method consists of mainly four modules including morpheme normalization, phrase-break detection, morpheme to phoneme conversion and phoneme connectivity check.

The morpheme normalization is to replace non-Korean symbols into standard Korean graphemes. The phrase-break detector assigns phrase breaks using part-of-speech (POS) information. In the morpheme-to-phoneme conversion module, each morpheme in the phrase is converted into phonetic patterns by looking up the morpheme phonetic pattern dictionary which contains candidate phonological changes in boundaries of the morphemes. Graphemes within a morpheme are grouped into CCV patterns and converted into phonemes by the CCV conversion rules. The phoneme connectivity table supports grammaticality checking of the adjacent two phonetic morphemes.

In the experiments with a corpus of 4,973 sentences, we achieved $99.9 \%$ of the graphemeto-phoneme conversion performance and $97.5 \%$ of the sentence conversion performance. The full Korean TTS system is now being implemented using this conversion method.
\end{abstract}

\section{Introduction}

During the past few years, remarkable improvements have been made for high-quality textto-speech systems (van Santen et al., 1997). One of the enduring problems in developing high-quality text-to-speech system is accurate grapheme-to-phoneme conversion (Divay and Vitale, 1997). It can be described as a function mapping the spelling of words to their phonetic symbols. Nevertheless, the function in some alphabetic languages needs some linguistic knowledge, especially morphological and phonological, but often also semantic knowledge.

In this paper, we present a new grapheme-tophoneme conversion method for unlimited vocabulary Korean TTS. The conversion method is divided into mainly four modules. Each module has its own linguistic knowledge. Phrasebreak detection module assigns phrase breaks onto part-of-speech sequences using morphological knowledge. Word-boundaries before and after phrase breaks should not be coarticulated. So, accurate phrase-break assignments are essential in high quality TTS systems. In the morpheme-to-phoneme conversion module, boundary graphemes of each morpheme in the phrase are converted to phonemes by applying phonetic patterns which contain possible phonological changes in the boundaries of morphemes. The patterns are designed using morphological and phonotactic knowledge. Graphemes within a morpheme are converted into phonemes by CCV (consonant consonant vowel) conversion rules which are automatically extracted from a corpus. After all the conversions, phoneme connectivity table supports the grammaticality of the adjacency of two phonetic morphemes. This grammaticality comes from Korean phonology rules.

This paper is organized as follows. Section 2 briefly explains the characteristics of spoken Korean for general readers. Section 3 and 4 introduces our grapheme-to-phoneme conversion method based on morphological and phonological knowledge of Korean. Section 5 shows experiment results to demonstrate the performance and Section 6 draws some conclusions. 


\section{Features of Spoken Korean}

This section briefly explains the linguistic characteristics of spoken Korean before describing the architecture.

A Korean word (called eojeol) consists of more than one morpheme with clear-cut morpheme boundaries (Korean is an agglutinative language). Korean is a postpositional language with many kinds of noun-endings, verb-endings, and prefinal verb-endings. These functional morphemes determine the noun's case roles, verb's aspect/tenses, modals, and modification relations between words. The unit of pause in speech (phrase break) is usually different from that in written text. No phonological change occur between these phrase breaks. Phonological changes can occur in a morpheme, between morphemes in a word, and even between words in a phrase break as described in the 30 general phonological rules for Korean(Korean Ministry of Education, 1995). These changes include consonant and vowel assimilation, dissimilation, insertion, deletion, and contraction. For example, noun "kag-ryo" pronounced as "kangnyo" (meaning "cabinet") is an example of phonological change within a morpheme. Noun plus noun-ending "such+gwa", in which "such" means "charcoal" and "gwa" means "and" in English, is sounded as "sudggwa", which is an example of the inter-morpheme phonological change. "Ta-seos gae", which means "five items", is sounded as "taseot ggae", in which phonological changes occur between words. In addition, phonological changes can occur conditionally on the morphotactic environments but also on phonotactic environments.

\section{Architecture of the Grapheme-to-Phoneme Converter}

Part-of-speech (POS) tagging is a basic step to the grapheme-to-phoneme conversion since phonological changes depend on morphotactic and phonotactic environments. The POS tagging system have to handle out-of-vocabulary (OOV) words for accurate grapheme-tophoneme conversion of unlimited vocabulary (Bechet and El-Beze, 1997). Figure 1 shows the architecture of our grapheme-to-phoneme converter integrated with the hybrid POS tagging system (Lee et al., 1997). The hybrid POS tagging system employs generalized OOV word handling mechanisms in the morphological analysis, and cascades statistical and rule-based approaches in the two-phase training architecture for POS disambiguation.

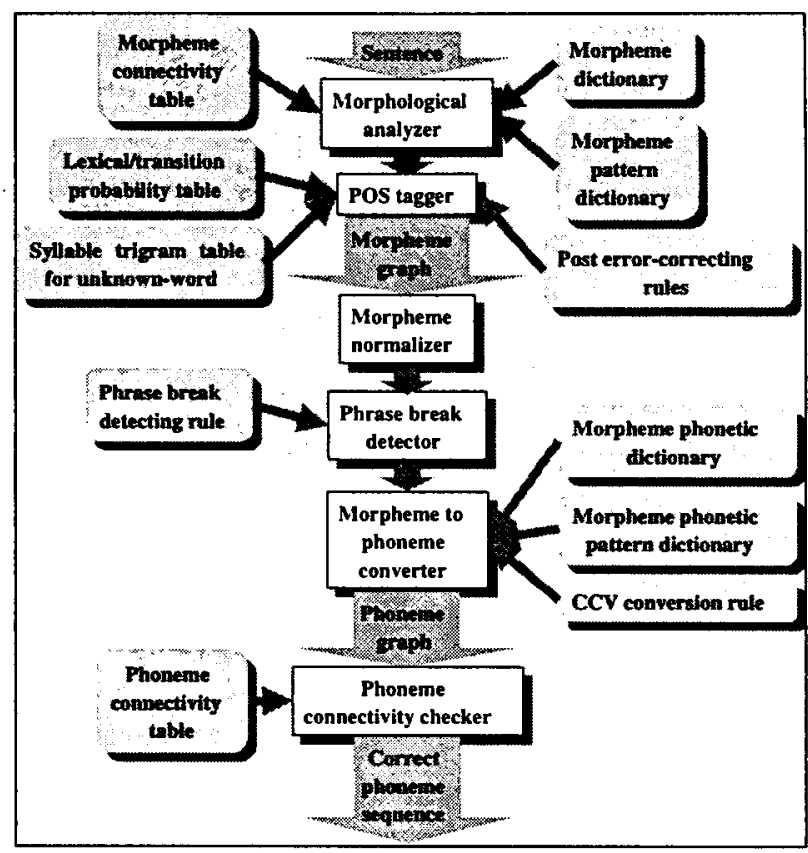

Figure 1: Architecture of the grapheme-tophoneme converter in TTS applications

Each morpheme tagged by the POS tagger is normalized by replacing non-Korean symbols by Korean graphemes to expand numbers, abbreviations, and acronyms. The phrase-break detector segments the POS sequences into several phrases according to phrase-break detection rules. In the phoneme converter, each morpheme in the phrase is converted into phoneme sequences by consulting the morpheme phonetic dictionary. The OOV morphemes which are not registered in the morpheme phonetic dictionary should be processed in two different ways. The graphemes in the morpheme boundary are converted into phonemes by consulting the morpheme phonetic pattern dictionary. The graphemes within morphemes are converted into phonemes according to CCV conversion rules. To model phoneme's connectablities between morpheme boundaries, the separate phoneme connectivity table encodes the phonological changes between the morpheme with their POS tags. Outputs of the graphemeto-phoneme converter, that is, phoneme se- 
quences of the input sentence, can be directly fed to the lower level signal processing module of TTS systems. Next section will give detail descriptions of each component of the graphemeto-phoneme converter. The hybrid POS tagging system will not be explained in this paper, and interested readers can see the reference (Lee et al., 1997).

\section{Component Descriptions of the Converter}

\subsection{Morpheme Normalization}

The normalization replaces non-Korean symbols by corresponding Korean graphemes. NonKorean symbols include numbers (e.g. 54, $12,5,400,4.2$ ), dates (e.g. 20/1/97, 20-Jan97), times (e.g. 12:46), scores (e.g. 74:64), mathematical expressions (e.g. $4+5,1 / 3$ ), telephone numbers, abbreviations (e.g. km, ha) and acronyms (e.g. UNESCO, OECD). Especially, acronyms have two types: spelled acronyms such as OECD and pronounced ones like a word such as UNESCO.

The numbers are converted into the corresponding Korean graphemes using deterministic finite automata. The dates, times, scores, expressions and telephone numbers are converted into equivalent graphemes using their formats and values. The abbreviations and acronyms are enrolled in the morpheme phonetic dictionary, and converted into the phonemes using the morpheme-to-phoneme conversion module.

\subsection{Phrase-Break Detection}

Phrase-break boundaries are important to the subsequent processing such as morphemeto-phoneme conversion and prosodic feature generation. Graphemes in phrase-break boundaries are not phonologically changed and sounded as their original corresponding phonemes in Korean.

A number of different algorithms have been suggested and implemented for phrase break detection (Black and Taylor, 1997). The simplest algorithm uses deterministic rules and more complicated algorithms can use syntactic knowledge and even semantic knowledge. We designed simple rules using break and POS tagged corpus. We found that, in Korean, the average length of phrases is 5.6 words and over $90 \%$ of breaks are after 6 different POS tags: conjunctive ending, auxiliary particle, case particle, other particle, adverb and adnominal ending. The phrase-break detector assigns breaks after these 6 POS tags considering the length of phrases.

\subsection{Morpheme-to-Phoneme Conversion}

The morphemes registered in the morpheme phonetic dictionary can be directly converted into phonemes by consulting the dictionary entries. However, separate method to process the OOV morphemes which are not registered in the dictionary is necessary. We developed a new method as shown Figure 2.

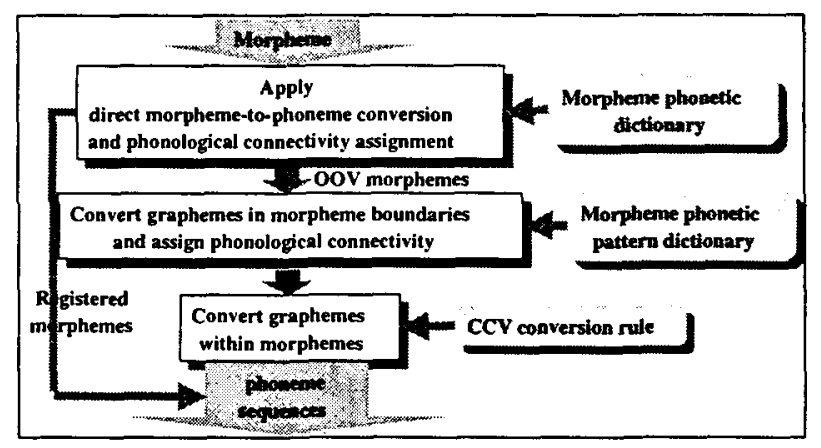

Figure 2: Morpheme-to-phoneme conversion for unlimited vocabularies

The morpheme phonetic dictionary contains POS tag, morpheme, phoneme connectivity (left and right) and phoneme sequence for each entry. We try to register minimum number of morpheme in the dictionary. So it contains only the morphemes which are difficult to process using the next OOV morpheme conversion modules. Table 1 shows example entries for the common noun "pang-gabs", meaning "price of a room" in hotel reservation dialogs. The common noun "pang-gabs" can be pronounced as "pang-ggam", "pang-ggab" or "pang-ggabss" according to first phoneme of the adjacent morphemes.

To handle the OOV morphemes, morpheme phonetic pattern dictionary is developed to contain all the general patterns of Korean POS tags, morphemes, phoneme connectivity and phoneme sequences. Boundary phonemes of the OOV morphemes can be converted to their candidate phonemes, and the phonological connectivity for them can be acquired by consulting this morpheme phonetic pattern dictionary. 
Table 1: Example entries of the morpheme phonetic dictionary

\begin{tabular}{|c|c|c|c|c|}
\hline POS tag & morpheme & phoneme sequence & left connectivity & right connectivity \\
\hline common noun & pang-gabs & pang-ggam & 'p' no change & 'bs' changed to ' $\mathrm{m}$ ' \\
\hline common noun & pang-gabs & pang-ggab & 'p' no change & 'bs' changed to ' $\mathrm{b}$ ' \\
\hline common noun & pang-gabs & pang-ggabss & 'p' no change & 'bs' changed to 'bss' \\
\hline
\end{tabular}

Table 2: Example entries of morpheme phonetic pattern dictionary

\begin{tabular}{|c|c|c|c|c|}
\hline POS tag & morpheme & phoneme sequence & left connectivity & right connectivity \\
\hline irregular verb & $\mathrm{t} * \mathrm{~d}$ & $\mathrm{tt} * \mathrm{n}$ & ' $\mathrm{t}$ ' changed to ' $\mathrm{tt}$ ' & 'd' changed to ' $\mathrm{n}$ ' \\
\hline irregular verb & $\mathrm{t} * \mathrm{Z}$ & $\mathrm{t} t * \mathrm{Z}$ & ' $\mathrm{t}$ ' changed to ' $\mathrm{t} \mathrm{t}$ ' & no change \\
\hline irregular verb & $\mathrm{Y} * \mathrm{~d}$ & $\mathrm{Y} * \mathrm{n}$ & no change & 'd' changed to ' $\mathrm{n}$ ' \\
\hline irregular verb & $\mathrm{Y} * \mathrm{Z}$ & $\mathrm{Y} * \mathrm{Z}$ & no change & no change \\
\hline
\end{tabular}

Example entries corresponding to the irregular verb "teud", meaning "hear", are shown in $\mathrm{Ta}$ ble 2. Meta characters, ' $\mathrm{Z}$ ', ' $\mathrm{Y}$ ', 'V', '*' designate single consonant, consonant except silence phoneme, vowel, any character sequence with variable length in the order. The table shows that the first grapheme ' $t$ ' can be phonologically changed to ' $t \mathrm{t}$ ' according to the last phoneme of the preceding morpheme (left connectivity), and the last grapheme 'd' can be phonologically changed to ' $n$ ' according to the first phoneme of the following morpheme(right connectivity). The morpheme phonetic pattern dictionary contains similar 1,992 entries to model the general phonological rules for Korean.

The graphemes within a morpheme for OOV morphemes are converted into phonemes using the CCV conversion rules. The CCV conversion rules are the mapping rules between grapheme to phoneme in character tri-gram forms which are in the order of consonant(C) consonant(C) vowel(V) spanning two consecutive syllables. The CCV rules are designed and automatically learned from a corpus reflecting the following Korean phonological facts.

- Korean is a syllable-base language, i.e., Korean syllable is the basic unit of the graphemes and consists of first consonant, vowel and final consonant (CVC).

- The number of possible consonants for each syllable can be varied in graphemeto-phoneme conversion.
- The number of vowels for each syllable is not changed.

- Phonological changes of the first consonant are only affected by the final consonant of the preceding syllable and the following vowel of the same syllable.

- Phonological changes of the final consonant are only affected by the first consonant of the following syllable.

- Phonological changes of the vowel are not affected by the following consonant.

The boundary graphemes of the OOV morphemes are phonologically changed according to the POS tag and the boundary graphemes of the preceding and following morphemes. On the other hand, the inner grapheme conversion is not affected by the POS tag, but only by the adjacent graphemes within the same morpheme. The CCV conversion rules can model the fact easily, but the conventional CC conversion rules (Park and Kwon, 1995) cannot model the influence of the vowels.

\subsection{Phoneme Connectivity Check}

To verify the boundary phonemes' connectablity to one another, the separate phoneme connectivity table encodes the phonologically connectable pair of each morpheme which has phonologically changed boundary graphemes. This phoneme connectivity table indicates the grammatical sound combinations in Korean 
phonology using the defined left and right connectivity information.

The morpheme-to-phoneme conversion can generate a lot of phoneme sequence candidates for single morpheme. We put the whole phoneme sequence candidates in a phoneme graph where a correct phoneme sequence path can be selected for input sentence. The phoneme connectivity check performs this selection and prunes the ungrammatical phoneme sequences in the graph.

\section{Implementation and Experiment Results}

We implemented simple phrase-break detection rules from break and POS tagged corpus collected from recording and transcribing broadcasting news. The rules reflect the fact that average length of phrases in Korean is 5.6 words and over $90 \%$ of breaks are after 6 specific POS tags, described in the texts.

We constructed a 1,992 entry morpheme phonetic pattern dictionary for OOV morpheme processing using standard Korean phonological rules. The morpheme phonetic dictionary was constructed for only the morphemes that are difficult to handle with these standard rules. The two dictionaries are indexed using POS tag and morpheme pattern for fast access. To model the boundary phonemes' connectablity to one another, the phoneme connectivity table encodes 626 pair of phonologically connectable morphemes.

The 2030 entry rule set for CCV conversion was automatically learned from phonetically transcribed 9,773 sentences. The independent phonetically transcribed 4,973 sentences are used to test the performance of the grapheme-tophoneme conversion. Of the 4,973 sentences, only $2.5 \%$ are incorrectly processed (120 sentences out of 4,973), and only $0.1 \%$ of the graphemes in the sentences are actually incorrectly converted.

\section{Conclusions}

This paper presents a new grapheme-tophoneme conversion method using phoneme connectivity and $\mathrm{CCV}$ conversion rules for unlimited vocabulary Korean TTS. For the efficient conversion, new ideas of morpheme phonetic and morpheme phonetic pattern dictio- nary are invented and the system demonstrates remarkable conversion performance for the unlimited vocabulary texts. Our main contributions include presenting the morphologically and phonologically conditioned conversion model which is essential for morphologically and phonologically complex agglutinative languages. The other contribution is the grapheme-to-phoneme conversion model combined with the declarative phonological rule which is well suited to the given task. We also designed new CCV unit of grapheme-tophoneme conversion for unlimited vocabulary task. The experiments show that graphemeto-phoneme conversion performance is $\mathbf{9 7 . 5 \%}$ in sentence conversion, and $99.9 \%$ in each grapheme conversion. We are now working on incorporating this grapheme-to-phoneme conversion into the developing TTS systems.

\section{References}

F. Bechet and M. El-Beze. 1997. Automatic assignment of part-of-speech to out-ofvocabulary words for text-to-speech processing. In Proceedings of the EUROSPEECH '97, pages 983-986.

Alan W. Black and Paul Taylor. 1997. Assigning phrase breaks from part-of-speech sequences. In Proceedings of the $E U$ ROSPEECH '97, pages 995-998.

Michel Divay and Anthony J. Vitale. 1997. Algorithms for grapheme-phoneme translation for English and French: Applications. Computational Linguistics, 23(4).

Korean Ministry of Education. 1995. Korean Rule Collections. Taehan Publishers. (in Korean).

Geunbae Lee, Jeongwon Cha, and Jong-Hyeok Lee. 1997. Hybrid POS tagging with generalized unknown-word handling. In Proceedings of the IRAL '97, pages 43-50.

S.H. Park and H.C. Kwon. 1995. Implementation to phonological alteration module for a Korean text-to-speech. In Proceedigns of the $4_{\text {th }}$ conference on Korean and Korean information processing. (in Korean).

Jan P.H. van Santen, Richard W. Sproat, Joseph P. Olive, and Julia Hirschberg. 1997. Progress in Speech Synthesis. SpringerVerlag. 